\title{
Vibration-Based Detection of Loosened Bolts on Pipes Attached to Bridges
}

\author{
Kobayashi Daiki ${ }^{1, a,{ }^{*}}$, Ikeguchi Yuta ${ }^{1, b}$, Nakagawa Masafumi ${ }^{1, c}$ \\ and Aratake Atsushi ${ }^{1, d}$
}

${ }^{1}$ NTT Corporation, 1-7-1 Hanabatake, Tsukuba, Ibaraki, Japan

adaiki.kobayashi.dh@hco.ntt.co.jp, byuuta.ikeguchi.da@hco.ntt.co.jp, cmasafumi.nakagawa.dr@hco.ntt.co.jp, datsushi.aratake.hf@hco.ntt.co.jp

Keywords: Civil Engineering, Vibration-Based Detection, Loosened Bolts, Dynamic Analysis, Pipeline

\begin{abstract}
U-bolts are critical points of maintenance in pipeline facilities supported by girders of bridges. However, it is difficult to detect loosened U-bolts by visual inspection. To avoid this problem, we have developed a vibration-based method to detect loosening of bolts based on measurements of actual equipment. First, the vibration of a communication pipeline attached to a road bridge was measured when vehicles passed over it. During the measurements, the U-bolts were set in tightened and loosened states. We found that the frequency changes between the tightened and loosened states was too small for practical use. On the other hand, a strong spectrum in the high frequency region (above $80 \mathrm{~Hz}$ ) appeared only in the tightened state. Next, a model facility was built to simulate a communication pipeline attached to bridge. A hammering test and modal and frequency response analyses were performed by using the finite element method (FEM). The results suggested that high frequency peaks appearing only in the tightened U-bolt are due to the higher rate of force transfer of the natural frequency from the platform. In addition, the pipe is strongly fixed when the U-bolt is tightened, which may lead to stronger high order modes that cause complex deformation of the pipe. The conventional method is to detect loosening of bolts from changes in the natural frequency. However, in actual equipment, confirming the absence of a high-frequency spectrum is more suitable for detecting loosened bolts.
\end{abstract}

\section{Introduction}

The pipelines of communication, electricity and gas infrastructure facilities are essential equipment of society. It is expensive to build underground tunnels, so pipelines are usually attached to girders of road bridges when they cross bodies of water or other roads. Some telecommunications and power pipelines are set on support structures of girders and secured by U-bolts. The U-bolts are critical maintenance points because of the risk of falling parts. Loose bolts are difficult to detect by visual inspection, so the development of an alternative method is desired.

Conventional methods to detecting loose bolts in civil engineering equipment are embedding sensors in bolts, vision-based, and vibration analysis. Sensors embedded in bolts can measure the tightening force $[1,2]$. Although this method is very accurate, the replacement cost of such infrastructure equipment in use is high. The vision-based method $[3,4]$ detects the rotation of the bolt heads from the difference between the initial and observed images. This method is cheaper than embedding sensors in the bolts, but it requires the bridge to be closed in order to take photographs. In this case, scaffolding has to be erected or drones have to be used because there are many blind spots under the girders where the pipes are installed. On the other hand, vibration-based methods $[5,6]$ may be cheaper than these methods for bolt detection. This is 
because dynamic measurements have been possible with optical fibers [7]. In this method, the optical fiber in the communication pipes acts as a dynamic sensor. This method could eliminate the need for on-site inspections and improve inspection quality.

The relationship between vibration and bolt loosening needs to be clarified in order to make fiber optic monitoring practical. He and Zhu [5] reported natural frequency changes in bolts attached to a pipe flange when the bolts were tightened and loosened. Razi et al. [6] devised an energy-based damage index by using empirical mode decomposition of bolts attached to a flange. The target applications of these studies are flanges, not U-bolts. In addition, they tested only models, and their applicability to actual facilities was not examined. Therefore, in this study, we measured U-bolts in actual facilities. On the basis of our results, we propose an optimum looseness detection method for U-bolts of pipelines.

\section{Actual Equipment Measurements}

Method

The measurement target was a communication pipeline attached to a road bridge in Japan (Fig. 1). The main girder of the bridge is made of concrete, and the length is $16 \mathrm{~m}$. The main girder has supporting metal to attach the pipeline. The pipes are made of rigid polyvinyl chloride and bonded by U-bolts. An accelerometer was installed at the position shown in Fig. 2. Measuring continued during the daytime to obtain the acceleration when vehicles passed over the bridge. The sampling rate was $1000 \mathrm{~Hz}$. The first half of the measurements were made with the U-bolt tightened, while the second half of the measurements were made with the U-bolt loosened. The acquired data were analyzed with a fast Fourier transform (FFT). The window function of the FFT was the exponential one shown as Eq. 1.

$$
w(x)=e^{\frac{x \log \beta}{N-1}} .
$$

Here, $w(x)$ is the weight of the window function, $x$ is time, $N$ is the number of data, and $\beta$ is the window function coefficient $(\beta=0.1)$.

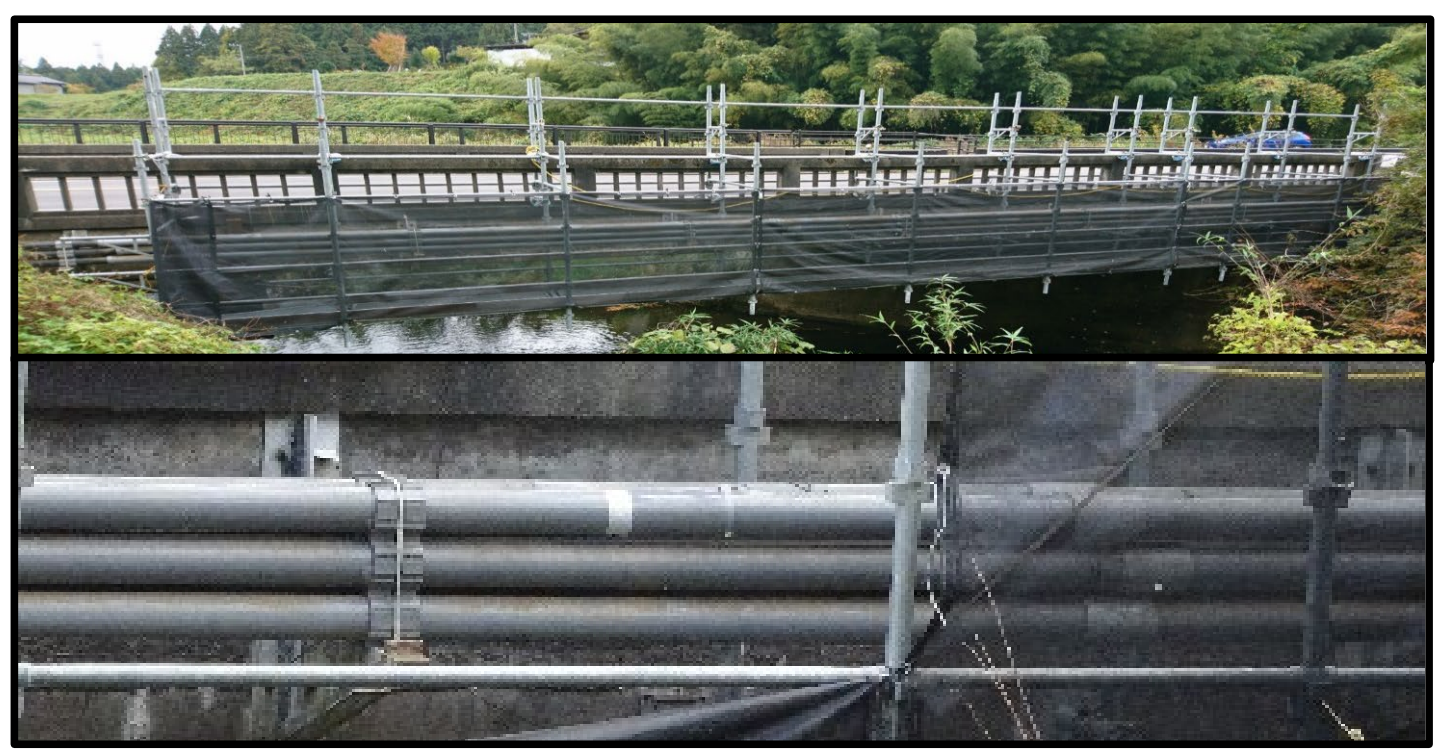

Figure 1. Measured communication pipes attached to the bridge [8] 


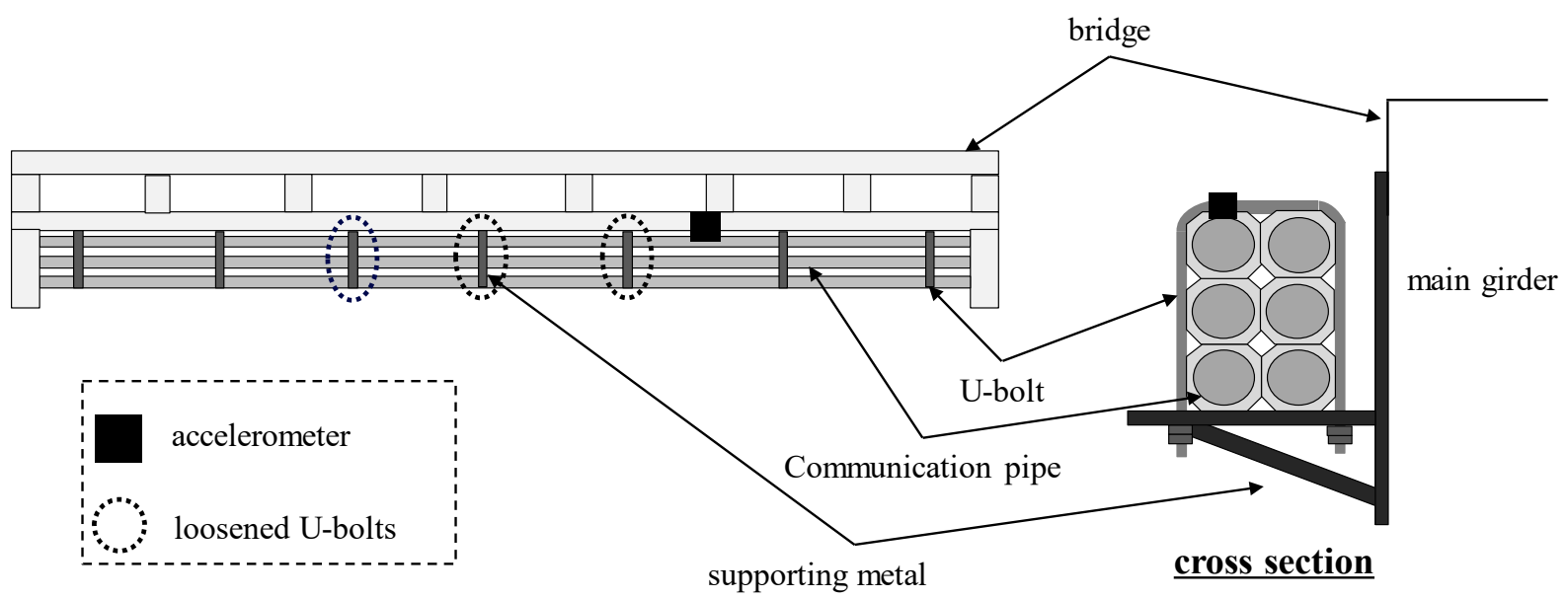

Figure 2. Overview of communication pipes attached to the bridge and setup of measurement

Results

The FFT data of the acceleration in the tightened and loosened states of the U-bolt are shown in Fig. 3. Nine waveforms for the tightened state and eight waveforms for the loosened state were obtained, which were additively averaged over the frequency domain. The frequency of the spectrum maximum was $15.0 \mathrm{~Hz}$ for the loosened state and $15.5 \mathrm{~Hz}$ for the tightened state, so the difference was $0.5 \mathrm{~Hz}$. In addition, spectrum peaks at $88 \mathrm{~Hz}$ and $144 \mathrm{~Hz}$ occurred only in the tightened state.

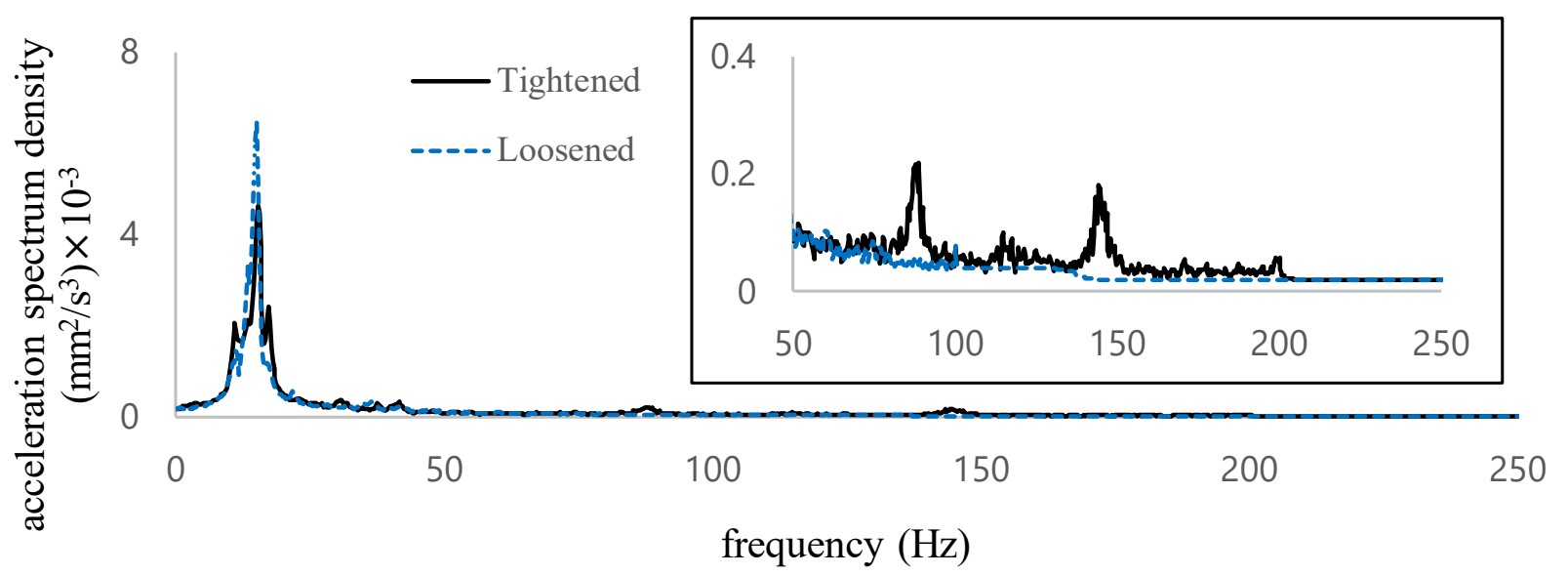

Figure 3. Measured acceleration in tightened and loosened states in actual equipment (Expanded view from 50 to $250 \mathrm{~Hz}$ is shown in the upper right corner)

\section{Model Facility Experiment}

A model of communication pipes was built for the hammering test and the simulation. The method of detecting loosened U-bolts is discussed by comparing the results of the model facility experiment and the measurements on the actual equipment. 


\section{Method}

A $5.5 \mathrm{~m}$ pipe made of rigid polyvinyl chloride was placed on a steel platform and bonded by U-bolts (Fig. 4). An accelerometer and a strain gauge for the plastic material were attached to the surface of the pipe at the center position of the support point. The accelerometer was set in the gravity direction, while the strain gauge was set in the bending direction of the pipe. The position shown in Fig. 4 was hit with a rubber hammer. The number of tests was 10 in both tightened and loosened states, and the results were additively averaged over the frequency domain.

Modal and frequency response analyses were performed on the experimental results. The geometry and conditions of the analyses are shown in Fig. 5, and the contact conditions between the pipe and the platform were set to bond. The platform bottoms of the 12 faces were fixed, and the damping ratio was set to $1 \%$. The analysis software was Ansys Mechanical 2020 R1.

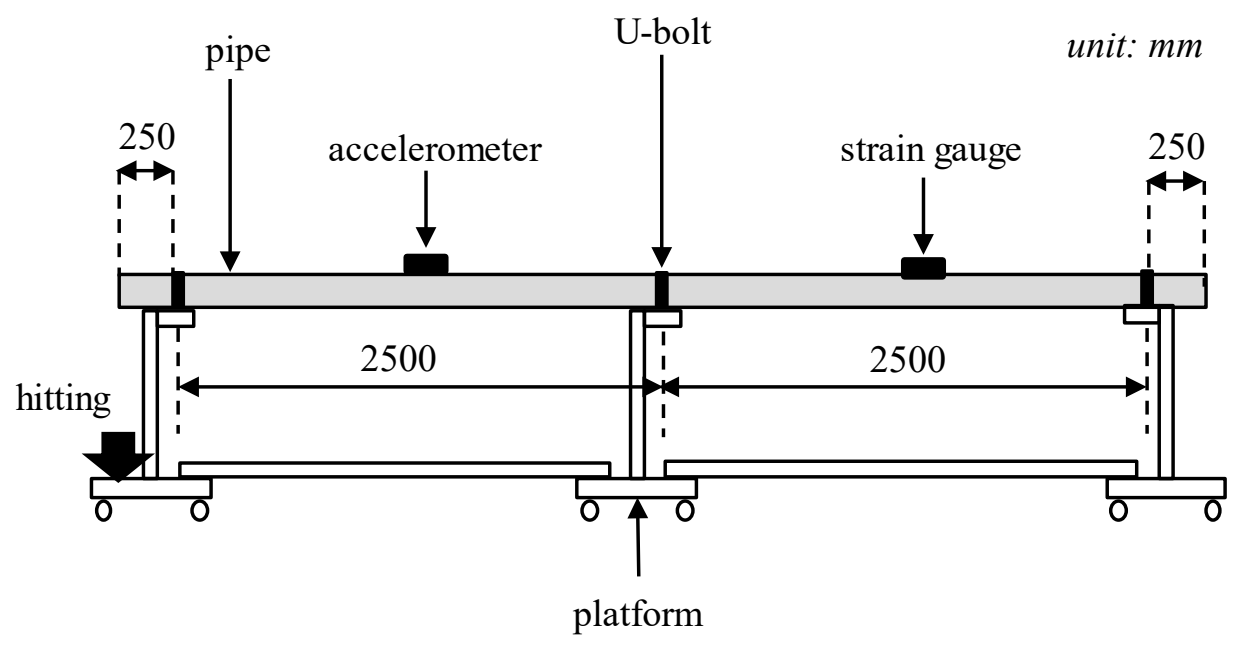

Figure 4. Overview of a model facility

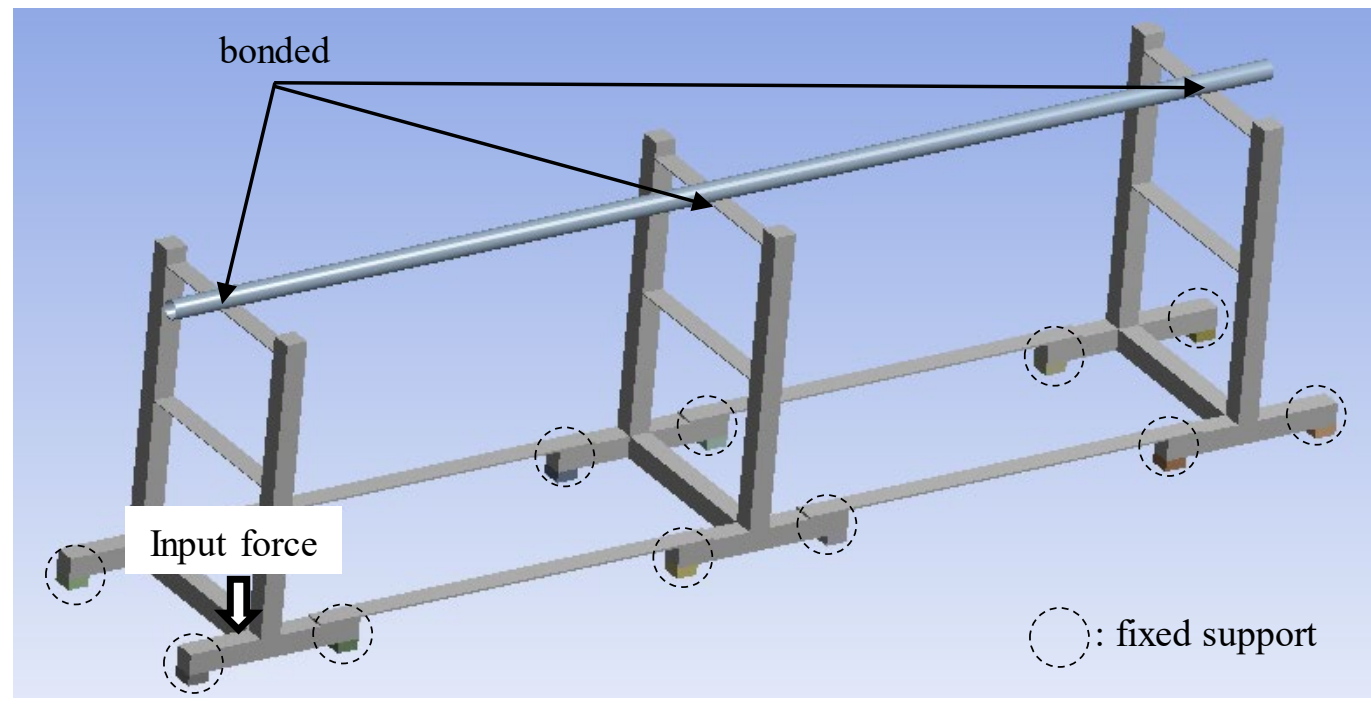

Figure 5. Overview of FEM model settings 
Results of Hammering Test

Fig. 6 shows the measured accelerations in the tightened and loosened states. Strong peaks occurred above $100 \mathrm{~Hz}$ when the U-bolt was in the tightened state only. The strain data are shown in Fig. 7. Multiple peaks for both tightened and loosened states were observed as in Figure 6. On the other hand, the intensity and frequency of the spectrum peaks above $100 \mathrm{~Hz}$ were smaller than those in Fig. 6.

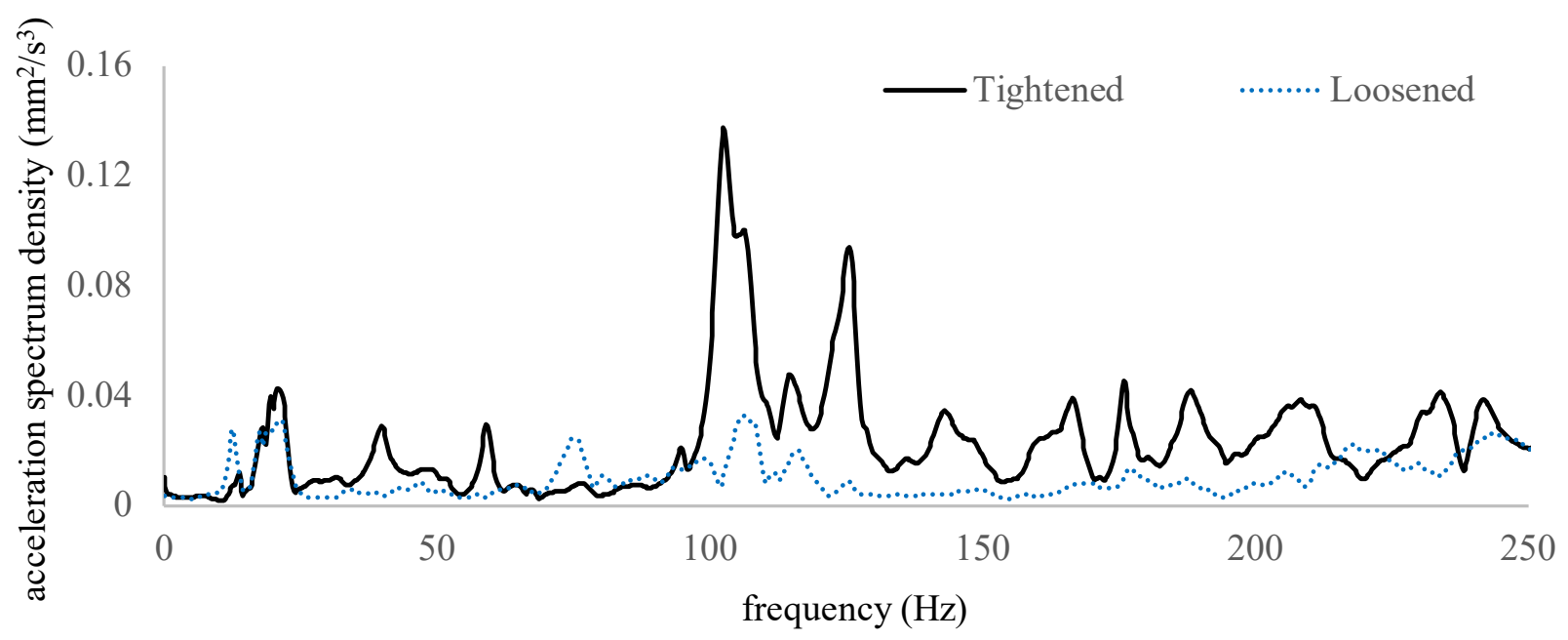

Figure 6. Measured acceleration for tightened and loosened U-bolts

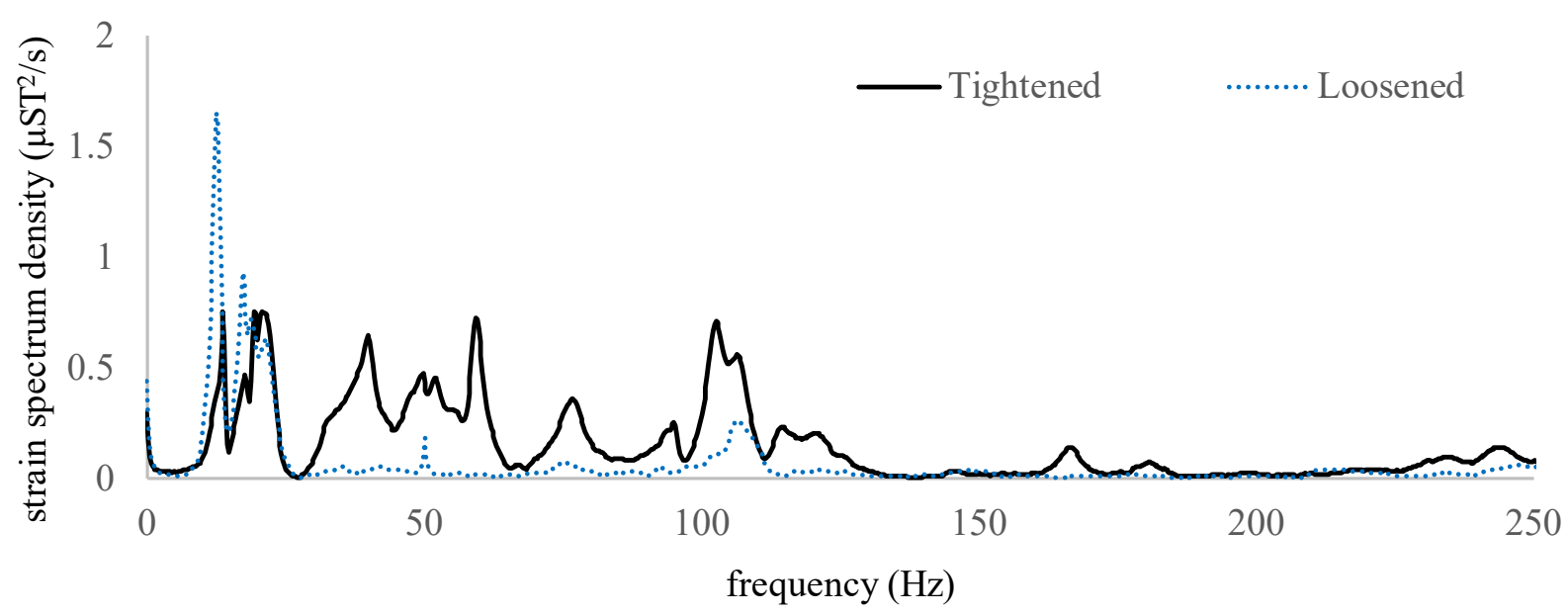

Figure 7. Measured strain in tightened and loosened U-bolts

Results of FEM Simulation

The results of the frequency response analysis are shown in Fig. 8. The normalized spectrum strength was defined as the relative strength at which the maximum spectrum strength $(20.8 \mathrm{~Hz})$ was 1 . Strong peaks existed around $21 \mathrm{~Hz}, 104 \mathrm{~Hz}$ and $114 \mathrm{~Hz}$, as in Fig. 6. In addition, there were many peaks above $100 \mathrm{~Hz}$, similar to Fig. 6. Therefore, it is considered that the simulation reproduced the experimental results. The results of the modal analysis are shown in Fig. 9. They 
show the relative deformations of the top four peaks above $100 \mathrm{~Hz}$ (A to D in Fig. 8). These deformations show complex modes such as multiple waves and cross-sectional deformation.

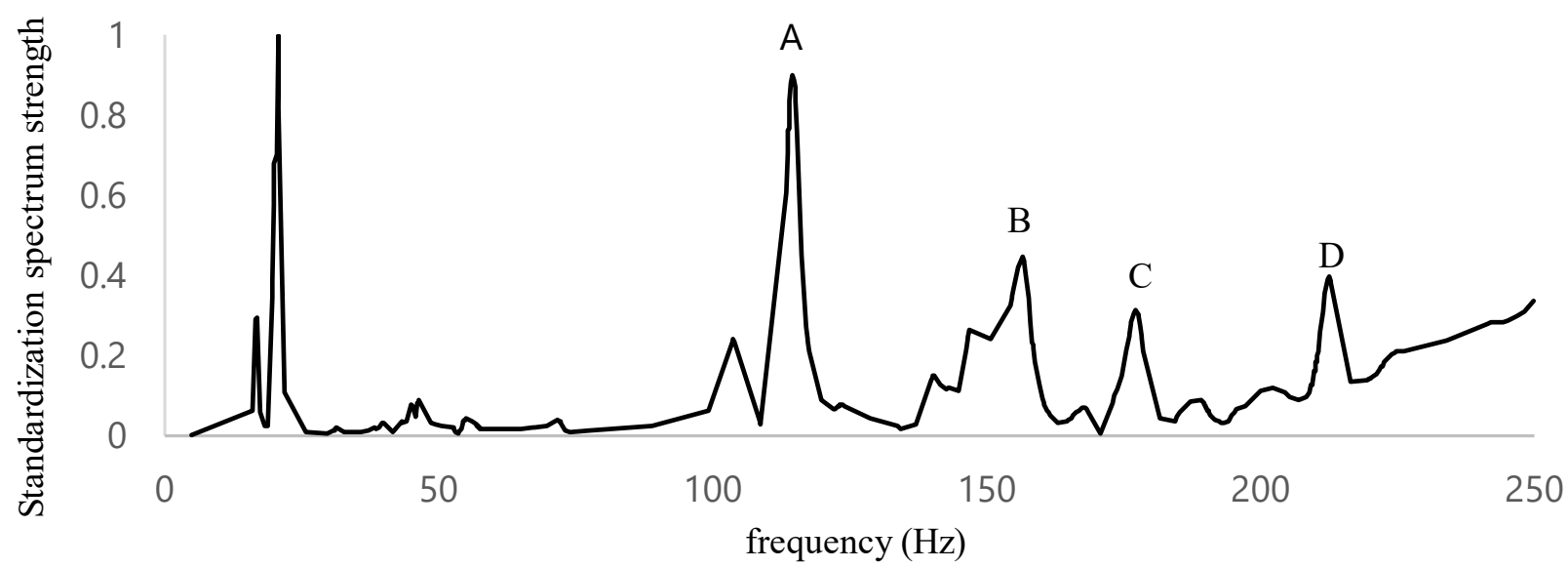

Figure 8. Results of frequency response analysis by FEM

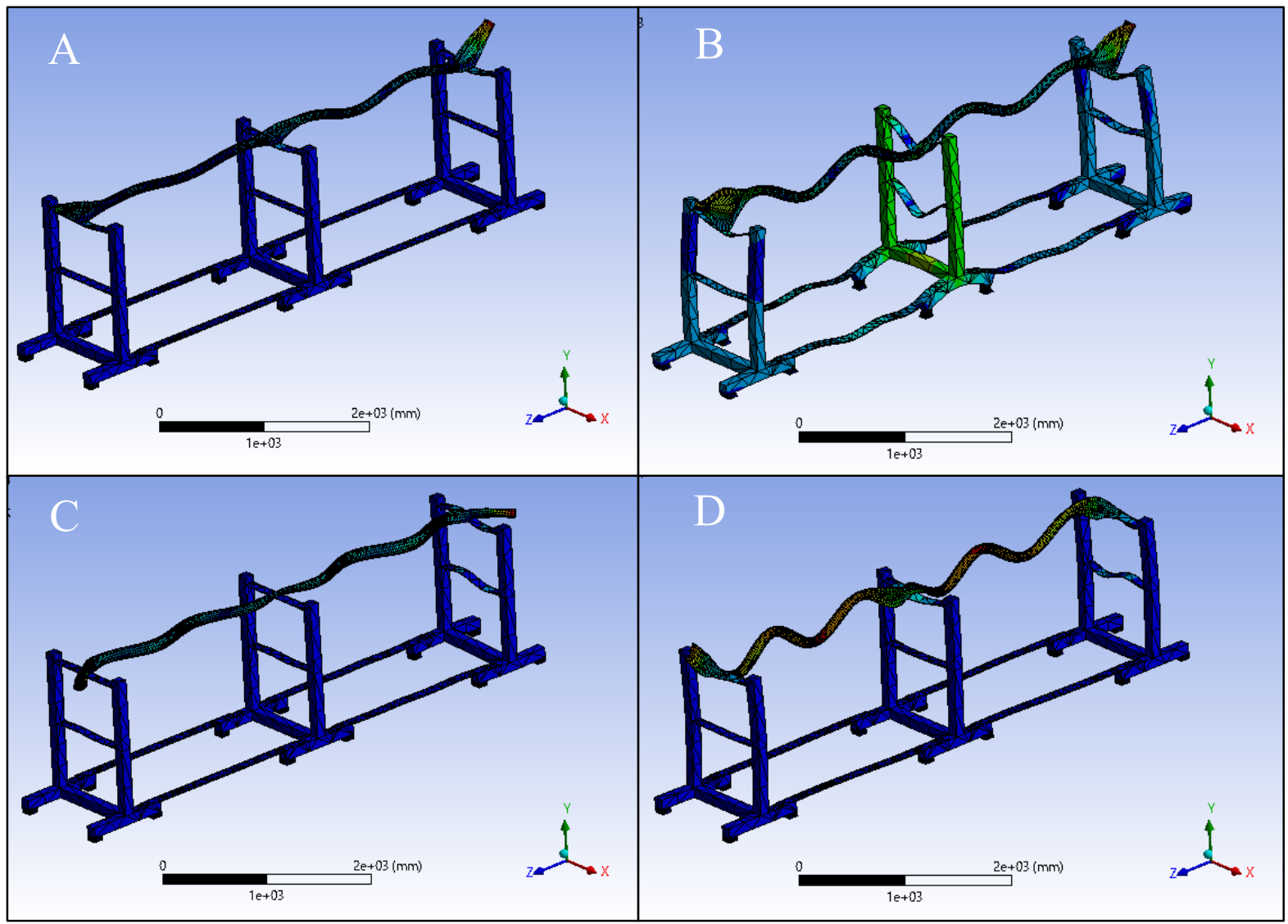

Figure 9. Results of modal analysis by FEM 


\section{Discussion}

Measuring the natural frequency shift is the simplest way to detect deteriorations such as loosened bolts. In the measurements of the actual facility shown in Fig. 3, the change in natural frequency was only $3 \%$. The natural frequency has a measurement error of 5-10\% [9], thus, this amount of change is too small for practical use. The change in the natural frequency is proportional to the square root of stiffness, so the natural frequency does not change sufficiently without a large stiffness change [10]. A previous study [5] reported the natural frequency change was over $15 \%$ for a bolt attached to the flange of a pipe. The bolts of flanges bear large stresses and contribute significantly to the stiffness of a pipe. However, U-bolts bear low stresses and have little impact on the stiffness of the pipe, so the change in natural frequency is small in this study.

Some peaks in the high-frequency region were observed in both the actual and model facilities only on the tightened state. Acceleration of the pipe was detected above $100 \mathrm{~Hz}$ (Fig. 6), but no bending strain was detected (Fig. 7) in that frequency region. These results may indicate that the pipe vibrated with no bending, because the rate of force transfer from the platform to the pipe increased when the U-bolt was tightened. In addition, the modal analysis of the model facility (Fig. 9) showed that the strong acceleration peaks above $100 \mathrm{~Hz}$ indicate complex deformation as a high-order mode of the pipe. Since the U-bolt is a node of the pipe, high order modes cannot appear when the fixing force of the node is weak.

From the above, we propose that confirming the absence of an acceleration response in the high frequency region is a more practical method than measuring the change in natural frequency. The remaining issue is a dynamic numerical simulation of the tightened and loosened states; it will improve the reliability of this method.

\section{Conclusion}

We examined a method of detecting loosened U-bolts in pipelines attached to bridges from vibrations. Actual measurements on a facility, a hammering test of model equipment, and a dynamic analysis were carried out. Our findings are as follows:

- Attempting to detect a change in the natural frequency, which is generally used for the detection of deterioration, was not practical, because the change in frequency was too small.

- Acceleration peaks in the high-frequency region (above $80 \mathrm{~Hz}$ ) occured only in the case of tightened U-bolts.

It was assumed that the peaks in the high-frequency region reflected the following phenomena:

- The U-bolt is a node of pipe deformation, so when the fixation with the U-bolt is weakened, the deformation is also reduced.

- The vibration from the platform is transmitted to the pipe. The rate of force transfer from the platform to the pipe is higher when the U-bolt is tightened.

We propose that confirming the absence of acceleration peaks in the high-freqency region is a practical method of detecting loosened U-bolts in pipelines. The remaining issue is the dynamic simulation of tightening and loosening. Completing the simulation will improve the reliability of this method.

\section{References}

[1] H. Yin, T. Wang, D. Yang, S. Liu, J. Shao, and Y. Li, A smart washer for bolt looseness monitoring based on piezoelectric active sensing method, Applied Sciences, vol. 6, no. 11, pp. 320-330, 2016. https://doi.org/10.3390/app6110320 
[2] N. Shimoi, C. H. Cuadra, H. Madokoro, and M. Saijo, Simple smart piezoelectric bolt sensor for structural monitoring of bridges, International Journal of Instrumentation Science, vol. 1, no. 5, pp. 78-83, 2013.

[3] X. Kong and J. Li, Image registration-based bolt loosening detection of steel joints, Sensors (Basel), vol. 18, no. 4, pp. 1000-1020, 2018.

[4] J. H. Park, T. C. Huynh, S. H. Choi, and J. T. Kim, Vision-based technique for bolt-loosening detection in wind turbine tower, Wind and Structures an International Journal, vol. 21, no. 6, pp. 709-726, 2015. https://doi.org/10.12989/was.2015.21.6.709

[5] K. He and W.D. Zhu, Detecting loosening of bolted connections in a pipeline using changes in natural frequencies, Proc. ASME 2011 Int. Des. Eng. Tech. Conf. Comput. Inf. Eng. Conf., 2011. https://doi.org/10.1115/DETC2011-47912

[6] P. Razi, R. A. Esmaeel, and F. Taheri, Improvement of a vibration-based damage detection approach for health monitoring of bolted flange joints in pipelines, Structural Health Monitoring, vol. 12, no. 3, pp. 207-224, 2013. https://doi.org/10.1177/1475921713479641

[7] Jousset, P., Reinsch, T., Ryberg, T., Blanck, H., Clarke, A., Aghayev, R., Hersir, G.P., Henninges, J., Weber, M., and Krawczyk, C.M. , Dynamic strain determination using fibre-optic cables allows imaging of seismological and structural features, Nature Communications, vol. 9, no. 1, pp. 1-11, 2018.

[8] D. Kobayashi, Y. Ikeguchi, C. Kazato, M. Nakagawa, and A. Aratake, Measurement of actual facilities for detecting loosening bolts attaching to bridge by vibration, Conference of Japanese Society of Civil Engineering, 2020. (In Japanese)

[9] W. Fan, and P. Qiao, Vibration-based damage identification methods: A review and comparative study, Structural Health Monitoring, vol. 10, no. 1, pp. 83-111, 2011. https://doi.org/10.1177/1475921710365419

[10] O.S. Salawu, Detection of structural damage through changes in frequency: A review. Engineering Structures, vol. 19, no. 9, pp. 718-723, 1997.

https://doi.org/10.1016/S0141-0296(96)00149-6 